\title{
The Line-of-Sight Depth of Populous Clusters in the Small Magellanic Cloud
}

\author{
Hugh H. Crowl ${ }^{1}$ and Ata Sarajedini ${ }^{2,3}$ \\ Astronomy Department, Wesleyan University, Middletown, CT 06459 \\ hugh@astro.yale.edu; ata@astro.ufl.edu \\ Andrés E. Piatti \\ Observatorio Astroómico, Laprida 854, 5000, Córdoba, Argentina \\ andres@mail.oac.uncor.edu \\ Doug Geisler \\ Grupo de Astronomia, Departmento de Fisica, Universidad de Concepción, Casilla 160-C, \\ Concepción, Chile \\ doug@kukita.cfm.udec.cl \\ Eduardo Bica \\ Departamento de Astronomia, Instituto de Fisica, UFRGS, CP 15051, 91501-970, Porto \\ Alegre, RS, Brazil \\ bica@if.ufrgs.br
}

\footnotetext{
${ }^{1}$ Current Address: Astronomy Department, Yale University, P. O. Box 208101, New Haven, CT 06520

${ }^{2}$ Guest User, Canadian Astronomy Data Centre, which is operated by the Dominion Astrophysical Observatory for the National Research Council of Canada's Herzberg Institute of Astrophysics.

${ }^{3}$ Current address: University of Florida, Department of Astronomy, Gainesville, FL 32611
} 
Juan J. Clariá

Observatorio Astroómico, Laprida 854, 5000, Córdoba, Argentina

claria@mail.oac.uncor.edu

and

João F. C. Santos, Jr.

Departamento de Fisica, ICEx, Universidade Federal de Minas Gerais, CP 702, 30123-970

Belo Horizonte, MG, Brazil

jsantos@fisica.ufmg.br

Received __; accepted _ 


\begin{abstract}
We present an analysis of age, metal abundance, and positional data on populous clusters in the Small Magellanic Cloud (SMC) with the ultimate aim of determining the line-of-sight (LOS) depth of the SMC using these clusters as proxies. Our dataset contains 12 objects and is limited to clusters with the highest quality data for which the ages and abundances are best known and can be placed on an internally consistent scale. We have analyzed the variation of the clusters' properties with position on the sky and with line-of-sight depth. Based on this analysis, we draw the following conclusions. 1) The observational data indicates that the eastern side of the SMC (facing the LMC) contains younger and more metal-rich clusters as compared with the western side. This is not a strong correlation because our dataset of clusters is necessarily limited, but it is suggestive and warrants further study. 2) Depending on how the reddening is computed to our clusters, we find a mean distance modulus that ranges from $(m-M)_{0}=18.71 \pm 0.06$ to $(m-M)_{0}=18.82 \pm 0.05$. 3) The intrinsic $\pm 1-\sigma$ line-of-sight depth of the SMC populous clusters in our study is between $\sim 6 \mathrm{kpc}$ and $\sim 12 \mathrm{kpc}$ depending primarily on whether we adopt the Burstein \& Heiles reddenings or those from Schlegel et al. 4) Viewing the SMC as a triaxial galaxy with the Declination, Right Ascension, and LOS depth as the three axes, we find axial ratios of approximately 1:2:4. Taken together, these conclusions largely agree with those of previous investigators and serve to underscore the utility of populous star clusters as probes of the structure of the Small Magellanic Cloud.
\end{abstract}

Subject headings: Clusters: Globular, SMC 


\section{Introduction}

The effect of interactions/mergers on the star formation histories of galaxies has become a field of intense activity (e.g. Carlson et al. 1998; Whitmore et al. 1999; Elmegreen et al. 2000). While many investigators have studied distant galaxy systems as a means of addressing this question, the Milky Way / Large Magellanic / Small Magellanic Cloud system provides a nearby example that is especially profitable scientifically (e.g. Gardiner 1999). This is because the relative proximity of these galaxies allows us to perform detailed age and abundance studies of individual field and cluster stars. Given the possibility that

the Milky Way and other spiral galaxies formed via the accretion/merger of dwarf satellite galaxies (Searle \& Zinn 1978; Côté et al. 2000), understanding the interplay between dynamical interactions and star formation is important in unlocking the secrets of galaxy formation.

One of the first pieces of evidence for the occurrence of dynamical interactions in the Milky Way/LMC/SMC system was the discovery of the Magellanic Stream by Wannier \& Wrixon (1972). Welch et al. (1987), Westerlund (1990), Bica \& Schmitt (1995) and Bica et al. (1999) discuss evidence for interactions between the Clouds based on the spatial distributions of stellar population tracers, such as the star clusters, associations, and emission nebulae. The recent work of Kunkel et al. (2000) reviews the formation of the Magellanic Stream and the interactions between the Large and Small Magellanic Clouds in detail. There is a fair amount of evidence, both from simulations (Murai \& Fujimoto 1980) and observations (Mathewson et al. 1988; Hatzidimitriou et al. 1989a; Gardiner \& Hawkins 1991) that, as a result of these interactions, the SMC may extend beyond its tidal radius. Some authors (Mathewson 1984; Mathewson et al. 1986) have even suggested that the SMC has split into two components: the Mini Magellanic Cloud (MMC) and the SMC Remnant. In a contrasting view, Welch et al. (1987) utilize infrared observations of cepheids 
to assert that the spatial extent of the SMC is well within its tidal radius. One of the most important pieces of evidence in determining the validity of these claims is the overall line of sight (LOS) depth of the Small Magellanic Cloud (SMC).

The most extensive series of papers dealing with the LOS depth of the SMC are those by Hatzidimitriou et al. (1989a), Hatzidimitriou et al. (1989b), Gardiner \& Hawkins (1991), and Gardiner \& Hatzidimitriou (1992). These authors studied the depth of the SMC using the magnitude spread of the horizontal branch/red clump (HB/RC) stars among the SMC field population. Their photographic survey, augmented by CCD observations of select fields, was quite extensive, covering 48.5 square degrees centered on the SMC. The basic premise of their investigation was that the magnitude of the HB/RC stars is mainly affected by distance and photometric errors but minimally influenced by the physical properties of the stars themselves, such as metallicity and age. Based on this assumption, they conclude that, not only is the SMC significantly dispersed in the LOS direction, but that the amount of dispersion varies with position on the sky. For example, the northeastern region located more than $2 \mathrm{kpc}$ from the optical center suffers the greatest LOS dispersion with an average LOS depth of $17 \mathrm{kpc}$ and a maximum of $23 \mathrm{kpc}$. In contrast, the analogous region located in the southwest displays a depth that is $\sim 10 \mathrm{kpc}$ shallower on average. In addition, most of the areas to the north and northwest of the optical center display a depth of between 4 and $9 \mathrm{kpc}$.

In the years since these landmark studies, there has been some controversy about the age and metallicity sensitivity of the red clump absolute magnitude. The reader is referred to the papers by Paczyński \& Stanek (1998), Udalski (1998), Cole (1998), Sarajedini (1999), and Girardi \& Salaris (2000) for some perspective on this question. The degree of sensitivity could have a significant effect on the derived LOS depth of the SMC based on the magnitude of the red clump. One way to explore the extent of this effect is to use 
observations of red clumps in SMC star clusters where we can define the age and metallicity far more precisely than in field star populations.

In order to explore the LOS depth of the SMC and the effect of assumptions concerning the variation of the red clump absolute magnitude, we have studied the distances to twelve SMC populous clusters that possess red clumps. These clusters are located all across the face of the SMC and span over three degrees in declination and almost one hour in right ascension. There are also several clusters near the right ascension of the optical center of the SMC $\left(\alpha_{2000}=0^{h} 52^{m} 45^{s}, \delta_{2000}=-72^{\circ} 49^{\prime} 43^{\prime \prime}\right)$. We will determine distances to these clusters assuming first that the sensitivity of the RC to age and metallicity is negligible, and then that the sensitivity is significant and is well-described by theoretical models. In Section 2, we will outline the sources from which the data were drawn and discuss the observational quantities obtained from the data. Section 3 is concerned with the theoretical isochrones and their calibration. Section 4 presents a discussion of our findings with regard to the depth of the SMC, as well as correlations between various cluster parameters. Finally, Section 5 presents the results and conclusions.

\section{Observational Data}

The observational data for this study, twelve SMC populous clusters spanning a range of ages and metallicities but all possessing predominantly red horizontal branches, are of three different pedigrees. Seven of the clusters (Lindsay 113, Kron 3, NGC 339, NGC 416, NGC 361, Lindsay 1, NGC 121) are taken from Mighell et al. (1998). In that work, these seven intermediate metallicity clusters $(-1.7 \lesssim[F e / H] \lesssim-1.2)$ were studied in order to better understand the star formation and chemical enrichment history of the Small Magellanic Cloud. Three of the clusters (Kron 28, Lindsay 38, Kron 44) are taken from the paper by Piatti et al. (2001). These data were obtained in the $C$ and $T_{1}$ filters of the 
Washington photometric system using the $0.9 \mathrm{~m}$ telescope at Cerro Tololo Inter-American Observatory. The observational data for the remaining two clusters (NGC 411 and NGC 152) come from archival Hubble Space Telescope / Wide Field Planetary Camera 2 images. These observations, taken as part of a snapshot survey of LMC/SMC clusters (Rich et al. $2000)$, consist of one image in the F450W $(\sim \mathrm{B})$ filter and another in the F555W $(\sim \mathrm{V})$ filter for each cluster. ${ }^{4}$ The observational data for these clusters are listed in Table 1 . In order to visualize the positions of the clusters within the field population of the SMC, Figure 1 shows a mosaic of Digitized Sky Survey images downloaded from Skyview ${ }^{5}$ This figure illustrates that, even though our dataset of clusters is relatively small, they cover a substantial portion of the SMC's spatial extent. We note that our cluster database is limited by the available resources. In particular, there is a serious dearth of published CCD observations for SMC star clusters. Nonetheless, as the database of SMC cluster observations increases, the techniques we have utilized in the present work will continue to be important as a probe of the SMC's three-dimensional structure.

\footnotetext{
${ }^{4}$ We had hoped to add NGC 419 to our dataset of SMC clusters. However, the CMD of 'NGC 419' presented by Rich et al. (2000) is actually that of NGC 411 observed at a different roll angle than the ostensive NGC 411 CMD. It is therefore not surprising that Rich et al. (2000) find NGC 411 and 'NGC 419' to have identical ages.

${ }^{5}$ SkyView was developed and is maintained under NASA ADP Grant NAS5-32068 with P.I. Thomas A. McGlynn under the auspices of the High Energy Astrophysics Science Archives Research Center (HEASARC) at the GSFC Laboratory for High Energy Astrophysics.
} 


\subsection{Reduction of HST Observations}

The standard image processing steps (de-biasing, flat-fielding, creating a bad pixel mask, etc) were performed by the Canadian Astronomy Data Centre ${ }^{6}$. The data reduction procedure was similar to that employed by Sarajedini (1998) and the reader is referred to that paper for more details. ${ }^{7}$ Using DAOPHOT II (Stetson 1994), we measured the magnitude of all detected profiles on each CCD (PC1, WF2, WF3, and WF4) within a radius of 2 pixels. Aperture corrections to a 0.5 " radius (10.87 PC1 pixels, 5 WF pixels) were then determined using 30 to 50 of the brightest uncrowded stars on each frame. With the aperture corrections applied, the F450W and F555W photometry was matched to form colors and transformed to the standard system of Holtzman et al. (1995). A $12 \%$ charge transfer efficiency correction in the $\mathrm{y}$-axis direction was applied following the recommendations of Holtzman et al. (1995) for observations taken at an operating temperature of $-76^{\circ} \mathrm{C}$. Using this reduction procedure, Sarajedini (1998) found good agreement between his HST/WFPC2 photometry for the LMC cluster NGC 2193 and ground-based photometry for the same cluster from Da Costa et al. (1987).

\footnotetext{
${ }^{6}$ Canadian Astronomy Data Centre is operated by the Dominion Astrophysical Observatory for the National Research Council of Canada's Herzberg Institute of Astrophysics.

${ }^{7}$ While these observations were reduced in the work of Rich et al. (2000), one of the most important concerns in the present study is internal consistancy, so we have re-reduced these images in the same way as the other HST clusters. Note that our results do not vary significantly from those of Rich et al. (2000).
} 


\subsection{Red Clump Magnitude}

In the case of the Mighell et al. (1998) clusters, the peak V magnitude of the red clump has already been computed and appears in their Table $9{ }^{8}$ For the remaining two sets of data (Piatti et al. 2001 and the HST photometry), color magnitude diagrams were first constructed as shown in Figures 2 through 5. On each of these diagrams, a box was drawn encompassing the location of the red clump in each cluster. The median $V$ magnitude of the stars in that box was taken to be representative of the peak $V$ magnitude of the red clump $(V(R C))$. The median was used to minimize the effect of outliers on $V(R C)$. Errors in the red clump magnitude were calculated from the standard error about the median. For NGC 411 and NGC 152, an additional error of 0.05 mag has been added due to uncertainties in the absolute photometric scale (see Mighell et al. 1998). These apparent magnitudes are provided in Table 2.

In the case of NGC 411 and NGC 152, there may be a concern that the measured red clump magnitude of the cluster has been unduly influenced by the red clump of the SMC field surrounding each cluster. Keeping in mind that the cluster was centered in the planetary camera (PC1), it is reasonable to assume that the cluster stars, and thus the cluster red clump, will be most prominent in the PC1 image. Indeed, we find this to be the case both for NGC 152 (Figure 3) and NGC 411 (Figure 4), where more red clump stars per unit area ${ }^{9}$ are present in the planetary camera as compared with the wide field chips. We find that the value of $V(R C)$ for the wide field cameras is identical to that of PC1. Therefore, we can safely combine the CMDs of all of the frames (Figure 5) and determine

\footnotetext{
${ }^{8}$ It should be noted that the value of $I_{0}(R C)$ for Lindsay 113 is incorrect in Table 5 of Udalski (1998). Instead of 18.33, the value of $I_{0}(R C)$ should be 18.13 .

${ }^{9}$ Since the planetary camera has $\sim 25 \%$ the field of view of the other cameras, with all other things being equal, one would expect $\sim 25 \%$ as many stars.
} 
$V(R C)$ from the composite CMD.

As mentioned above, the observations of Piatti et al. (2001) were obtained using the Washington (Canterna 1976) photometric system. The Washington system consists of four different filters, some of which roughly approximate those in the Johnson-Kron-Cousins UBVRI system. Geisler (1996) has provided robust relations that can be used to convert between these two systems. We begin by transforming the $T_{1}$ magnitude of the red clump to Johnson $V$ using the coefficients in Table 4 of Geisler (1996). In order to do this, it was first necessary to transform the $\mathrm{RC} T_{1}$ magnitude into an $R$ magnitude. From there, we converted the $C-T_{1}$ color into a $V-R$ color. Having $R$ and $V-R$, we then simply determined the $V$ magnitude of the red clump. The errors in $V(R C)$ were calculated by transforming $T_{1}+1 \sigma_{T_{1}}$ and $T_{1}-1 \sigma_{T_{1}}$ into $V$ magnitudes and using those values to deduce the standard error about the median in $V$. In addition to these standard errors, there was an uncertainty introduced when the transformation was applied. These errors, which are presented in Table 4 of Geisler (1996), were added in quadrature to the standard errors about the median to obtain the total uncertainty in $V(R C)$.

We note that the clusters K28, L38, and K44 are located near the optical center of the SMC where the field star contamination is high. As a result, we have used the field subtracted photometry for these clusters derived by Piatti et al. (2001) and plotted in Figure 2. We have used the median to compute the red clump magnitude because it is relatively insensitive to which subset of cluster stars are used to locate the red clump.

\subsection{Interstellar Extinction}

We seek an approach that yields internally consistent reddenings for each of our clusters. As such, we have avoided using individual literature values for each cluster opting 
instead to utilize the reddening maps of Burstein \& Heiles (1982) and Schlegel et al. (1998). First, the traditional Burstein \& Heiles (1982) (hereafter BH) reddening maps are based primarily on HI column densities at declinations appropriate for the SMC. Second, the more modern COBE/DIRBE reddening maps of Schlegel et al. (1998) (hereafter SFD) provide all-sky extinction values determined from the infrared emission of interstellar dust. In this study, we will make use of both of these options making sure to point out significant differences in the analysis as they occur. To determine $A_{V}$, we have adopted $A_{V}=3.1 \cdot E(B-V)$ (Cardelli et al. 1989; Schlegel et al. 1998). Since no formal errors are given by the maps, the errors in the reddening are taken to be $10 \%$ of the reddening value itself.

\subsection{Ages and Metallicities}

Because the cluster age and metallicity could influence the luminosity of the red clump (Sarajedini 1999; Girardi et al. 2000), we also require knowledge of these quantities in order to determine the absolute red clump magnitude $\left(M_{V}(R C)\right)$ and thus the distance to each cluster. Published values were adopted for these quantities as given in Table 2. The majority are from the previously mentioned Mighell et al. (1998) work, where a large amount of our photometric data originated. The metallicities for these clusters were determined using the simultaneous reddening and metallicity (SRM) method of Sarajedini (1994) as well as a new calibration of the RGB slope as a function of metallicity, both of which are on the Zinn \& West (1984) abundance scale. The ages of the clusters in Mighell et al. (1998) were determined via the technique described by Sarajedini, Lee, \& Lee (1995), which uses the difference between the RGB color and the age-sensitive HB color, 
corroborated by isochrone comparisons to the main sequence turnoff regions. ${ }^{10}$ Their age scale assumes that Lindsay 1 is 9 Gyr old (Olszewski et al. 1996).

In the case of the three clusters from the work of Piatti et al. (2001), the metal abundances are a result of comparing the observed RGBs of the clusters with the standard RGBs in the Washington photometric system set up by Geisler \& Sarajedini (1999). Note that Kron 28 has a different metal abundance depending on whether Piatti et al. (2001) adopt the Burstein \& Heiles (1982) reddening maps or those of Schlegel et al. (1998) (see Section 4). This is indicated in Table 2 by listing two numbers with the latter in parenthesis. The ages of these clusters rely upon the magnitude difference between the red clump and the main sequence turnoff along with a calibration provided by Geisler et al. (1997) that relates this magnitude difference to age. Again, the metal abundances are on the scale of Zinn \& West (1984), and the age scale assumes 9 Gyr for Lindsay 1.

Lastly, we consider the clusters whose photometry was measured specifically for the present study. The metallicity of NGC 411 has been determined in Alves \& Sarajedini (1999) using the same SRM method (Sarajedini 1994) as Mighell et al. (1998). Because NGC 411 is significantly younger than the other clusters, however, Alves \& Sarajedini (1999) use a formulation for Galactic open clusters from Noriega-Mendoza \& Ruelas-Mayorga (1997), which yields an abundance that is in good agreement with other determinations. To estimate the metal abundance of NGC 152, we utilize the same technique employed by

\footnotetext{
${ }^{10}$ Mighell et al. (1998) correctly point out that age estimates for their clusters based only on the main sequence turnoff would be subject to significant uncertainty. This is because the short exposure archival HST observations on which their study is based result in unacceptably large photometric scatter in the region of the turnoff. If Mighell et al. had relied solely on the magnitude and color of the turnoff to determine the cluster ages, they would not have attained the high level of precision that ultimately resulted from their analysis.
} 
Alves \& Sarajedini (1999) in the case of NGC 411. We begin by fitting a polynomial to the RGB. This is then input into the SRM method as modified by Noriega-Mendoza \& Ruelas-Mayorga (1997), which yields a metallicity of $[\mathrm{Fe} / \mathrm{H}]=-0.94 \pm 0.15$ for NGC 152 . The age of NGC 411 was determined by Alves \& Sarajedini (1999) using the Bertelli et al. (1994) isochrones fitted to the main sequence turnoff. They find that NGC 411 is $1.4 \pm 0.2$ Gyr old. Using these same isochrones, Mighell et al. (1998) find an age of $9 \pm 1$ Gyr for Lindsay 1. Because our age scale is based on Lindsay 1 being 9 Gyr old, no correction has been applied to the age of NGC 411. For the age of NGC 152, we again employ the technique of Alves \& Sarajedini (1999) and fit the Bertelli et al. (1994) isochrones to our CMD which, like the case of NGC 411, yields an age of $1.4 \pm 0.2$ Gyr for NGC 152 .

To close out this section, we seek to compare our cluster ages and metallicities with recent compilations in the literature. In particular, for the 7 clusters in common with the study of Udalski (1998), we find $\Delta[F e / H]=-0.05 \pm 0.05$ dex and $\Delta$ Age $=+0.1 \pm 0.3$ Gyr, where the differences have been computed in the sense (Us-Udalski). Similarly, for the 7 clusters in common with Da Costa \& Hatzidimitriou (1998), we find $\Delta[F e / H]=-0.04 \pm 0.05$ dex and $\Delta$ Age $=-0.5 \pm 0.5$ Gyr. These differences are not statistically significant and thus provide support for the validity of our cluster ages and abundances.

\section{Theoretical Isochrones}

In order to determine the variation of the red clump absolute magnitude $\left[M_{V}(R C)\right]$ with age and metal abundance, we utilize the theoretical horizontal branch models of Girardi et al. (2000) (see also Girardi \& Salaris (2000)), which span the metallicity range from $Z=0.0004$ to $Z=0.030$; additional models with $Z=0.0001$ were kindly provided by Leo Girardi. There is now abundant evidence supporting the validity of these theoretical models for the purpose of the present analysis. Not only is there the work of Sarajedini (1999) and 
Girardi et al. (2000) which compare the models to open cluster red clumps, but there is also the work of Girardi (1999) and Girardi \& Salaris (2000) which compare them to the red clumps of composite populations such as the Galactic bulge and the LMC. In addition, Castellani et al. (2000) present a comparison of the Girardi et al. models with those of other authors.

Figure 6 shows a spline fit to the median absolute magnitude of the red clump as a function of metallicity and age. Isochrones for ages of (top to bottom) 2.0, 2.5, 5.0, 7.0, 7.9, 10.0, and 12.6 Gyr are shown by the solid lines. An isochrone for an age of 1 Gyr is shown by the dashed line. For ages younger than $\sim 2$ Gyr, the isochrones become fainter again before reversing course and brightening for ages younger than $\sim 1.2$ Gyr. We utilize isochrones as young as 1 Gyr in our calculations. In order to calibrate the distance scale of the theoretical models to observational data, we rely upon $M_{V}(R C)$, age, and metallicity values for eight open clusters from Sarajedini (1999). In that work, the distance modulus to each open cluster was determined using main sequence fitting to M67, which was in turn fitted to the solar abundance Bertelli et al. (1994) isochrones. Utilizing the distance determined via this method, along with $V(R C)$, Sarajedini (1999) was able to compute $M_{V}(R C)$ for all of the clusters in his study. In the present analysis, we use the mean values of the eight open clusters from Sarajedini (1999) to calibrate the theoretical models. The average red clump absolute magnitude of the open clusters is $M_{V}(R C)=0.950 \pm 0.035$ at an average age of $2.68 \pm 0.75$ Gyr and an average metallicity of $-0.04 \pm 0.06$ dex as shown in Fig. 6. The theoretical values of $M_{V}(R C)$ from Girardi et al. were increased by +0.093 mag to bring them onto the distance scale of Sarajedini (1999). These calibrated models were then used to determine the absolute magnitude of the red clump for the twelve clusters in this work as described in the next section. 


\section{Results and Discussion}

Using the theoretical isochrones illustrated in Fig. 6, we are able to determine the absolute magnitude of the red clump $\left(M_{V}(R C)\right)$ for the twelve clusters in our sample. These absolute magnitudes, when coupled with the measured apparent magnitude of the red clump $(V(R C))$ and the interstellar extinction $(E(B-V))$, yield the distance to each cluster. The total error in the distance includes errors in the values of $M_{V}(R C), V(R C)$ and $A_{V}$.

The resultant distances are listed in Table 3 under different assumptions for the variation of $M_{V}(R C)$ (line 1 and 2 for each cluster) and the adopted reddenings (columns 3 and 4 for each cluster). We consider four distinct scenarios. 1) The absolute magnitude of the red clump is influenced by age and metallicity, as given in Fig. 6, coupled with the BH reddening values. 2) This is the same as (1) except that we adopt the SFD reddenings. 3) The absolute magnitude of the red clump is not influenced by age and metallicity, in which case we adopt the mean $M_{V}(R C)$ of our 12 clusters $\left(\left\langle M_{V}(R C)\right\rangle=0.56 \pm 0.04\right)$, along with the $\mathrm{BH}$ reddening values. 4) This is the same as (3) except that the SFD reddenings are used.

Given these cluster distances computed under various assumptions, we can calculate the mean distance modulus of our 12 clusters. Under assumptions (1) and (3) above, both of which use the $\mathrm{BH}$ reddenings, we find $(m-M)_{0}=18.82 \pm 0.05$, while assumption (2) and (4) yield $(m-M)_{0}=18.71 \pm 0.06$ based on the SFD reddenings. The quoted errors represent standard errors of the mean for the 12 clusters. It is important to reiterate that the distance scale used here is that established by Sarajedini (1999) based on main sequence fitting to open clusters (Section 3). Both of these distance moduli are consistent with the latest determination based on a combination of red clump stars, RR Lyraes, the tip of the RGB, and Cepheids presented by Udalski (2000). He finds $(m-M)_{0}=18.75 \pm 0.07$ 
for the SMC. This agreement underscores the fact that the distance scale which yields consistent results from these four indicators is in turn consistent with the open cluster scale of Sarajedini (1999).

With the line-of-sight distance to the clusters and accurate positions in right ascension and declination (Welch 1991), we are able to determine the distribution of these clusters in three dimensional space. While the small number of clusters available to us represents a limitation in studying the overall structure of the SMC, it does allow us to examine the principal focus of this work, which is the overall depth of the SMC (Section 4.2).

\subsection{Correlations Between Observed and Derived Quantities}

As an initial examination of the observational data, Fig. 7 shows the extinctioncorrected red clump magnitudes for our 12 clusters as a function of their metal abundances and ages. The former has been calculated using $V(R C)$ along with the extinction values inferred from both the $\mathrm{BH}$ and SFD reddening maps. Taking into account the selection effects and the small number of clusters, Fig. 7 displays no apparent trends.

We can also examine the behavior of the variation of cluster age and metal abundance as a function of position on the sky as shown in Fig. 8. Note that Kron 28 has been plotted with the metallicity inferred by its $\mathrm{BH}$ reddening. There are no definite trends apparent in Fig. 8, and, given the selection effects and the small number of clusters, it is difficult to unequivocally isolate a specific variation; however, the data do hint at a possible correlation. There is a tendency for the clusters to be younger and more metal-rich on the eastern side of the SMC (facing the LMC) as compared with the western side. This phenomenon was also noted by Gardiner \& Hatzidimitriou (1992); they linked it to the past interactions between the SMC and LMC. However, Gardiner \& Hatzidimitriou (1992) speculate that, 
instead of stimulating star formation in this region, the interaction with the LMC 'pulled out' this region from the SMC exposing its existing stellar populations (Piatti et al. 2001).

Given the cluster distances in Table 3, Fig. 9 illustrates the behavior of this distance with position on the sky. Here we have computed the angular offsets in Right Ascension and Declination from the optical center of the SMC (Sec. 1) and converted this to a linear distance using the distance to each cluster. Once again, the small number of clusters prevents us from making any definitive statements. However, it is important to point out that several previous studies based on the positions of SMC field stars - Hatzidimitriou et al. (1989b) for red clump stars, Mathewson et al. $(1986,1988)$ for Cepheids, and Kunkel et al. (2000) for carbon stars - have concluded that the northeastern portion of the SMC is closer to us while the southwestern region is farther away. In contrast, as shown in Fig. 9, the 12 SMC clusters studied herein display little or no evidence of this inclination. If this result is not simply due to the small number of clusters and continues to hold up as

more clusters are added to the database, it indicates that the SMC field stars do not share in the spatial properties of the clusters. The extant kinematical information (Da Costa \& Hatzidimitriou 1998) suggests that both of these components possess similar velocity characteristics, i.e. no significant rotation. In any case, there appears to be a paucity of observational data - both for the clusters and field stars - to allow an adequate treatment of this apparent discrepancy.

\subsection{The Depth of the Populous Clusters}

To estimate the line of sight depth of the SMC, we consider the standard deviation $\left(\sigma_{o b s}\right)$ of the cluster distances using the small-sample statistical formulae of Keeping (1962). We note that $\sigma_{o b s}$ is influenced by two factors: 1) The intrinsic depth of the clusters along the line of sight $\left(\sigma_{\text {int }}\right)$, and 2) the fact that each of our cluster distances is also affected by 
measurement errors $\left(\sigma_{\text {err }}\right)$. These two factors combine in quadrature to create the overall observed standard deviation:

$$
\sigma_{\text {obs }}^{2}=\sigma_{\text {int }}^{2}+\sigma_{\text {err }}^{2}
$$

The intrinsic standard deviation is then $\sqrt{\sigma_{o b s}^{2}-\sigma_{e r r}^{2}}$.

In Table 4, we present the results of this analysis under the four different assumptions outlined above. First, if the red clump magnitude is sensitive to age and abundance, then, using the curves illustrated in Fig. 6 and the $\mathrm{BH}$ reddenings $\left(M_{V}(R C)+\mathrm{BH}\right)$, we find an intrinsic $1-\sigma$ depth of $\sigma_{i n t}=3.2 \pm 0.7 \mathrm{kpc}$. On the other hand, if we adopt the SFD reddenings $\left(M_{V}(R C)+\mathrm{SFD}\right)$, a $1-\sigma$ depth of $\sigma_{i n t}=3.7 \pm 0.8 \mathrm{kpc}$ results. If the red clump magnitude is insensitive to age and metallicity, then, using the mean $M_{V}(R C)$ of our clusters along with the $\mathrm{BH}$ reddenings $\left(\left\langle M_{V}(R C)\right\rangle+\mathrm{BH}\right)$ produces an intrinsic SMC depth of $\sigma_{\text {int }}=3.9 \pm 0.8 \mathrm{kpc}$. This result is modified significantly if we instead utilize the SFD reddenings $\left(\left\langle M_{V}(R C)\right\rangle+\mathrm{SFD}\right)$, in which case $\sigma_{\text {int }}=6.0 \pm 1.2 \mathrm{kpc}$.

Therefore, regardless of which assumption we make for the absolute magnitude of the red clump, the statistically significant range of distances among the populous clusters indicates that the SMC does indeed exhibit a substantial extent in the LOS direction in agreement with several previous investigations. Using the language of Gardiner \& Hawkins (1991), our data indicate that the SMC has a mean "2- $\sigma$ depth" (i.e. $\pm 1 \sigma$ ) of between $\sim 6 \mathrm{kpc}$ and $\sim 12 \mathrm{kpc}$. This is consistent with the values quoted by Gardiner \& Hawkins (1991), which lie anywhere between 4 and $16 \mathrm{kpc}$ depending on which portion of the SMC one is observing.

There is one potentially important caveat to keep in mind when comparing our LOS depth results to those of Hatzidimitriou et al. (1989a) and Gardiner \& Hawkins (1991). These studies calculated the LOS depth by observing several relatively small field regions distributed around the SMC and using the height of the red clump (corrected for 
photometric errors) as a depth indicator. The overall depth is then the numerical average of these individual depths. Even though Fig. 9 does not suggest the existence of an inclination among the SMC clusters, if there is a significant global inclination to the SMC (field stars and clusters) relative to the line of sight, then our LOS depth as calculated using equation 1 will be systematically larger than those quoted by Hatzidimitriou et al. (1989a) and Gardiner \& Hawkins (1991).

To give the reader some 'perspective' on the distribution in space of the SMC clusters, Figs. 10a and b show a three-dimensional plot of the 12 star clusters in the present study under assumptions (1) and (2), respectively. Figures 9 and 10 suggest that the SMC appears to be a triaxial galaxy. Adopting the Declination, Right Ascension, and LOS depth as the three axes, we find axial ratios of approximately 1:2:4.

\section{Conclusions}

In this work, we have compiled published photometry for 12 Small Magellanic Cloud populous clusters that possess helium burning red clumps. After adopting metallicities on the Zinn \& West (1984) scale and ages on a scale where Lindsay 1 is 9 Gyr old, we have analyzed the variation of these properties with position on the sky and with line-of-sight depth. Based on this analysis, we draw the following conclusions.

1) The observational data indicate that the eastern side of the SMC (facing the LMC) contains younger and more metal-rich clusters as compared with the western side. This is not a strong correlation because our dataset of clusters is necessarily limited, but it is suggestive and warrants further study.

2) Using theoretical models describing the luminosity of the red clump and its variation with age and abundance zeropointed to the scale of main sequence fitting distances to Galactic 
open clusters, coupled with the Burstein \& Heiles (1982) reddening maps, we find a mean distance modulus for our 12 SMC clusters of $(m-M)_{0}=18.82 \pm 0.05$. If the Schlegel et al. (1998) reddenings are adopted instead, this is reduced to $(m-M)_{0}=18.71 \pm 0.06$. These results do not change significantly if the corrections to the Girardi et al. (2000) models are applied.

3) The intrinsic $\pm 1-\sigma$ line-of-sight depth of the SMC populous clusters in our study lies between $\sim 6 \mathrm{kpc}$ and $\sim 12 \mathrm{kpc}$ depending on assumptions regarding the variation of red clump luminosity and the amount of interstellar reddening.

4) Viewing the SMC as a triaxial galaxy with the Declination, Right Ascension, and LOS depth as the three axes, we find axial ratios of approximately 1:2:4.

Taken together, these conclusions largely agree with those of previous investigators and serve to underscore the utility of populous star clusters as probes of the structure of the Small Magellanic Cloud.

D.G. acknowledges financial support for this project received from CONICYT through Fondecyt grants 1000319 and 8000002, and by the Universidad de Concepción through research grant No. 99.011.025. 


\section{REFERENCES}

Alves, D. \& Sarajedini, A. 1999, ApJ, 511, 225

Bertelli, G., Bressan, A., Chiosi, C., Fagotto, F., \& Nasi, E., 1994, A\&AS, 106, 275

Bica, E., \& Schmitt, H. R. 1995, ApJS, 101, 41

Bica, E., Schmitt, H. R., Dutra, C. M., \& Oliveira, H. L. 1999, AJ, 117, 238

Burstein, D., \& Heiles, C. 1982, AJ, 87, 1165

Canterna, R. 1976, AJ, 81, 228

Cardelli, J. A., Clayton, G. C., \& Mathis, J. S. 1989, ApJ, 345, 245

Carlson, M. N., Holtzman, J. A., Watson, A. M., Grillmair, C. J., Mould, J. R., Ballester, G. E., Burrows, C. J., Clarke, J. T., Crisp, D., Evans, R. W., Gallagher, J. S., Griffiths, R. E., Hester, J. J., Hoessel, J. G., Scowen, P. A., Stapelfeldt, K. R., Trauger, J. T., \& Westphal, J. A. 1998, AJ, 115, 1778

Castellani, V., Degl'Innocenti, S., Girardi, L., Marconi, M., Prada Moroni, P. G., Weiss, A., A\&A, 354, 150

Cole, A. 1998, ApJ, 500, L137

Côté, P., Marzke, R.O., West, M.J., \& Minniti, D. 2000, ApJ, 533, 869

Da Costa, G. S., \& Hatzidimitriou, D. 1998, AJ, 115, 1934

Da Costa, G. S., King, C. R., \& Mould, J. R. 1987, ApJ, 321, 735

Elmegreen, B. G., Kaufman, M., Struck, C., Elmegreen, D. M., Brinks, E., Thomasson, M., Klaric, M., Levay, Z., English, J., Frattare, L. M., Bond, H. E., Christian, C. A., Hamilton, F., \& Noll, K. 2000, AJ, 120, 630 
Gardiner, L. T. 1999, in New Views of the Magellanic Clouds, IAU Symp. No. 190, (ASP: San Francisco) p. 480

Gardiner, L.T. \& Hawkins, M.R.S. 1991, MNRAS, 251, 174

Gardiner, L.T. \& Hatzidimitriou, D. 1992, MNRAS, 257, 195

Geisler, D. 1996, AJ, 111, 480

Geisler, D., \& Sarajedini, A. 1999, AJ, 117, 308

Geisler, D., Bica, E., Dottori, H., Clariá, J. J., Piatti, A. E., \& Santos, J. F. C., Jr. 1997, AJ, 114, 1920

Girardi, L. 1999, MNRAS, 308, 818

Girardi, L, Mermilliod, J.-C., Carraro, G. 2000, A\&A, 354, 892

Girardi, L., \& Salaris, M. 2000, MNRAS, in press (astro-ph/0007343)

Girardi, L., Bressan, A., Bertelli, G., \& Chiosi, C. 2000, A\&AS, 141, 371

Hatzidimitriou, D., Hawkins, M.R.S., Gyldenkerne, K. 1989a, MNRAS, 241, 645

Hatzidimitriou, D., Hawkins, M.R.S. 1989b, MNRAS, 241, 667

Holtzman, J.A., Burrows, C.J., Casertano, S., Hester, J.J., Trauger, J.T., Watson, A.M., \& Worthey, G. 1995, PASP, 107, 156

Keeping, E.S. 1962, Introduction to Statistical Inference, (Princeton: Van Nostrand)

Kunkel, W.E., Demers, S., \& Irwin, M.J. 2000, AJ, 119, 2789

Mathewson, D.S. 1984, Mercury, March-April, 57

Mathewson, D.S., Ford, V.L., Visvanathan, N. 1986, ApJ, 301, 664 
Mathewson, D.S., Ford, V.L., Visvanathan, N. 1988, ApJ, 333, 617

Mighell, K.J., Sarajedini, A., \& French, R.S. 1998, AJ, 116, 2395

Murai, T., \& Fujimoto, M. 1980, PASJ, 32, 581

Noriega-Mendoza, H., \& Ruelas-Mayorga, A. 1997, AJ, 113, 722

Olszewski, E. W., Suntzeff, N., \& Mateo, M. 1996, ARA\&A, 34, 511

Paczyński, B., \& Stanek, K. Z. 1998, ApJ, 494, L219

H.

Piatti, A.E., Santos Jr., J.F.C., Clariá, J.J., Bica, E., Sarajedini, A., Geisler, D. 2000, AJ, submitted

Rich, R.M., Shara, M., Fall, S.M., \& Zurek, D. 2000, AJ, 119, 197

Sarajedini, A. 1994, AJ, 107, 618

Sarajedini, A. 1998, AJ, 116, 738

Sarajedini, A. 1999, AJ, 118, 2321

Sarajedini, A., Lee, Y.-W., \& Lee, D.-H. 1995, ApJ, 450, 712

Searle, L. \& Zinn, R. 1978, ApJ, 225, 357

Schlegel, D.J., Finkbeiner, D.P., \& Davis, M. 1998, ApJ, 500, 525

Stetson, P.B. 1994, PASP, 106, 250

Udalski, A. 1998, AcA, 48, 383

Udalski, A. 2000, AcA, 50, 279 
Wannier, P. \& Wrixon, G.T. 1972, ApJ, 119, L119

Welch, D.L., McLaren, R.A., Madore, B.F., \& McAlary, C.W. 1987 ApJ, 321, 162

Welch, D.L. 1991, AJ, 101, 538

Westerlund, B. E. 1990, A\&ApR, 2, 29

Whitmore, B. C., Zhang, Q., Leitherer, C., Fall, S. M., Schweizer, F., \& Miller, B. W. 1999, AJ, 118, 1551

Zinn, R. J., \& West, M. J. 1984, ApJS, 55, 45

This manuscript was prepared with the AAS LATEX macros v5.0. 


\section{Figure Captions}

Fig. 1.- Mosaic of Digital Sky Survey images obtained from Skyview of the twelve clusters and their positions within the SMC.

Fig. 2.- Color Magnitude Diagrams of three SMC populous clusters with photometry in the Washington Photometric system from the work of Piatti et al. (2001). A box is drawn around the red clump of each cluster.

Fig. 3.- NGC 152 CMDs from the four individual CCDs. Notice the prominant red clump in planetary camera, which is less obvious in the three wide field images. A box is drawn around the cluster red clump in each panel.

Fig. 4. - Same as Fig. 3 except that the CMDs of NGC 411 are shown.

Fig. 5.- Combined Wide Field and Planetary Camera color magnitude diagrams of two SMC clusters from observations with the Hubble Space Telescope. A box is drawn around the red clump of each cluster.

Fig. 6. - The absolute red clump magnitude from the Girardi et al. (2000) isochrones zeropointed to the mean absolute magnitude of open cluster red clumps from the study of Sarajedini (1999) at their mean metallicities and ages as shown by the plotted data point. The models indicated by solid lines are for ages of (top to bottom) 2.0, 2.5, 5.0, 7.0, 7.9, 10.0, and 12.6 Gyr. The dashed line is for an age of 1 Gyr.

Fig. 7.- The variation of the extinction corrected $\mathrm{V}$ magnitude of the red clump as a function of cluster metallicity (left) and age (right). The upper panels show the variation when the Burstein \& Heiles (1982) reddenings are adopted while the lower panels how the result of adopting the Schlegel et al. (1998) reddenings. 
Fig. 8. - The variation of cluster age (bottom) and metal abundance (top) as a function of Right Ascension (left) and Declination (right).

Fig. 9.- (top) The distance of each cluster in our dataset as a function of its Right Ascension (top) and Declination (bottom) offset from the optical center of the SMC. The left panels show the result of adopting the Burstein \& Heiles (1982) reddenings while the right panels how the result of the Schlegel et al. (1998) reddenings. In the upper panels, east is to the left and in the lower panels, south is to the left.

Fig. 10.- (a) Three dimensional plot of SMC cluster positions assuming that the red clump magnitude is sensitive to age and abundance along with the ? reddenings. (b) Same as (a) except along a different line-of-sight. 
Table 1. HST Observation Log

\begin{tabular}{cccc}
\hline \hline \multirow{2}{*}{ Cluster } & Date & Filter & Exposure Time \\
& $(\mathrm{yy} / \mathrm{mm} / \mathrm{dd})$ & & $(\mathrm{s})$ \\
\hline \multirow{2}{*}{ NGC 411 } & $94 / 05 / 25$ & F450W & 400.0 \\
& $94 / 05 / 25$ & F555W & 200.0 \\
NGC 152 & $94 / 09 / 27$ & F450W & 300.0 \\
& $94 / 09 / 27$ & F555W & 160.0 \\
\hline
\end{tabular}


Table 2. SMC Cluster Properties

\begin{tabular}{|c|c|c|c|c|c|c|}
\hline Cluster & {$[\mathrm{Fe} / \mathrm{H}]$} & $\begin{array}{c}\text { Age } \\
\text { (Gyr) }\end{array}$ & Reference $^{a}$ & $V(R C)$ & $E(B-V)_{B H}^{b}$ & $E(B-V)_{S}^{b}$ \\
\hline NGC 411 & $-0.68 \pm 0.07$ & $1.4 \pm 0.2$ & 1 & $19.43 \pm 0.05$ & 0.03 & 0.12 \\
\hline NGC 152 & $-0.94 \pm 0.15$ & $1.4 \pm 0.2$ & 2 & $19.57 \pm 0.05$ & 0.03 & 0.05 \\
\hline Lindsay 113 & $-1.24 \pm 0.11$ & $5.3 \pm 1.3$ & 3 & $19.15 \pm 0.02$ & 0.02 & 0.05 \\
\hline Kron 3 & $-1.16 \pm 0.09$ & $6.0 \pm 1.3$ & 3 & $19.45 \pm 0.05$ & 0.02 & 0.03 \\
\hline NGC 339 & $-1.50 \pm 0.14$ & $6.3 \pm 1.3$ & 3 & $19.46 \pm 0.05$ & 0.03 & 0.05 \\
\hline NGC 416 & $-1.44 \pm 0.12$ & $6.9 \pm 1.1$ & 3 & $19.74 \pm 0.05$ & 0.04 & 0.12 \\
\hline NGC 361 & $-1.45 \pm 0.11$ & $8.1 \pm 1.2$ & 3 & $19.53 \pm 0.05$ & 0.05 & 0.13 \\
\hline Lindsay 1 & $-1.35 \pm 0.08$ & $9.0 \pm 1.0$ & 3 & $19.34 \pm 0.02$ & 0.01 & 0.03 \\
\hline NGC 121 & $-1.71 \pm 0.10$ & $11.9 \pm 1.3$ & 3 & $19.73 \pm 0.05$ & 0.04 & 0.03 \\
\hline Kron 28 & $-1.20(-1.45) \pm 0.13^{c}$ & $2.1 \pm 0.5$ & 4 & $19.33 \pm 0.05$ & 0.06 & 0.16 \\
\hline Lindsay 38 & $-1.65 \pm 0.12$ & $6.0 \pm 0.5$ & 4 & $19.56 \pm 0.05$ & 0.02 & 0.02 \\
\hline Kron 44 & $-1.10 \pm 0.11$ & $3.1 \pm 0.8$ & 4 & $19.52 \pm 0.05$ & 0.02 & 0.05 \\
\hline
\end{tabular}

a(1) Alves \& Sarajedini (1999); (2) This paper; (3) Mighell et al. (1998); (4) Piatti et al. (2001)

${ }^{b}$ An error of $10 \%$ has been assumed in the reddenings.

${ }^{\mathrm{c}}$ As determined by Piatti et al. (2000) adopting a reddening from Burstein \& Heiles (1982). The value in parenthesis is the result of adopting the Schlegel et al. (1998) reddening. 
Table 3. SMC Cluster Red Clump Distances

\begin{tabular}{|c|c|c|c|}
\hline Cluster & $M_{V}(R C)$ & $\begin{array}{c}\operatorname{Distance}(\mathrm{BH})^{a} \\
(\mathrm{kpc})\end{array}$ & $\begin{array}{c}\text { Distance }(\mathrm{SFD})^{b} \\
(\mathrm{kpc})\end{array}$ \\
\hline \multirow[t]{2}{*}{ NGC 411} & $0.63 \pm 0.16$ & $55.1 \pm 4.3$ & $48.5 \pm 3.8$ \\
\hline & $0.56 \pm 0.04$ & $56.9 \pm 1.6$ & $50.1 \pm 1.7$ \\
\hline \multirow[t]{2}{*}{ NGC 152} & $0.49 \pm 0.19$ & $62.7 \pm 5.7$ & $61.0 \pm 5.5$ \\
\hline & $0.56 \pm 0.04$ & $60.7 \pm 1.9$ & $59.0 \pm 1.8$ \\
\hline \multirow[t]{2}{*}{ Lindsay 113} & $0.57 \pm 0.07$ & $50.5 \pm 1.7$ & $48.4 \pm 1.7$ \\
\hline & $0.56 \pm 0.04$ & $50.8 \pm 1.1$ & $48.6 \pm 1.1$ \\
\hline \multirow[t]{2}{*}{ Kron 3} & $0.62 \pm 0.05$ & $56.7 \pm 1.9$ & $55.9 \pm 1.8$ \\
\hline & $0.56 \pm 0.04$ & $58.3 \pm 1.7$ & $57.5 \pm 1.7$ \\
\hline \multirow[t]{2}{*}{ NGC 339} & $0.57 \pm 0.06$ & $57.5 \pm 2.1$ & $55.8 \pm 2.0$ \\
\hline & $0.56 \pm 0.04$ & $57.7 \pm 1.7$ & $56.1 \pm 1.7$ \\
\hline \multirow[t]{2}{*}{ NGC 416} & $0.60 \pm 0.05$ & $63.6 \pm 2.1$ & $56.7 \pm 2.1$ \\
\hline & $0.56 \pm 0.04$ & $64.7 \pm 1.9$ & $57.8 \pm 2.0$ \\
\hline NGC 361 & $0.64 \pm 0.04$ & $55.8 \pm 1.7$ & $49.8 \pm 1.7$ \\
\hline
\end{tabular}


Table 3-Continued

\begin{tabular}{|c|c|c|c|}
\hline Cluster & $M_{V}(R C)$ & $\begin{array}{c}\text { Distance }(\mathrm{BH})^{a} \\
(\mathrm{kpc})\end{array}$ & $\begin{array}{c}\text { Distance }(\mathrm{SFD})^{b} \\
(\mathrm{kpc})\end{array}$ \\
\hline \multirow{3}{*}{ Lindsay 1} & $0.56 \pm 0.04$ & $57.9 \pm 1.7$ & $51.7 \pm 1.8$ \\
\hline & $0.68 \pm 0.03$ & $53.2 \pm 0.9$ & $51.7 \pm 0.9$ \\
\hline & $0.56 \pm 0.04$ & $56.2 \pm 1.1$ & $54.6 \pm 1.2$ \\
\hline \multirow[t]{2}{*}{ NGC 121} & $0.73 \pm 0.04$ & $59.6 \pm 1.8$ & $60.5 \pm 1.8$ \\
\hline & $0.56 \pm 0.04$ & $64.4 \pm 1.8$ & $65.4 \pm 1.9$ \\
\hline \multirow[t]{3}{*}{ Kron 28} & $0.27 \pm 0.12$ & $59.5 \pm 3.6$ & $54.8 \pm 3.3$ \\
\hline & $(0.14 \pm 0.11)^{c}$ & & \\
\hline & $0.56 \pm 0.04$ & $52.1 \pm 1.8$ & $45.2 \pm 1.7$ \\
\hline \multirow[t]{2}{*}{ Lindsay 38} & $0.51 \pm 0.03$ & $62.7 \pm 1.9$ & $62.7 \pm 1.9$ \\
\hline & $0.56 \pm 0.04$ & $61.3 \pm 1.9$ & $61.3 \pm 1.8$ \\
\hline \multirow[t]{2}{*}{ Kron 44} & $0.49 \pm 0.08$ & $62.2 \pm 2.7$ & $59.6 \pm 2.6$ \\
\hline & $0.56 \pm 0.04$ & $60.2 \pm 1.8$ & $57.7 \pm 1.8$ \\
\hline
\end{tabular}

${ }^{a}$ Calculated adopting the red clump absolute magnitudes in Column (2) and the Burstein \& Heiles (1982) reddenings listed in column 5 of Table 2.

${ }^{\mathrm{b}}$ Calculated adopting the red clump absolute magnitudes in Column 2 and the Schlegel et al. (1998) reddenings listed in column 6 of Table 2.

${ }^{\mathrm{c}}$ As determined from Fig. 6 adopting the metallicity from Piatti et al. (2000) derived assuming a reddening from Burstein \& Heiles (1982). 
Table 4. SMC Depth Results

\begin{tabular}{cccc}
\hline \hline Assumption & $\sigma_{\text {obs }}$ & $\sigma_{\text {err }}$ & $\sigma_{\text {int }}$ \\
& $\mathrm{kpc}$ & $(\mathrm{kpc})$ & $(\mathrm{kpc})$ \\
\hline & & & \\
1) $M_{V}(R C)+\mathrm{BH}$ & $4.1 \pm 0.8$ & $2.5 \pm 0.4$ & $3.2 \pm 1.0$ \\
2) $M_{V}(R C)+\mathrm{SFD}$ & $4.4 \pm 0.9$ & $2.4 \pm 0.4$ & $3.7 \pm 1.1$ \\
3) $\left\langle M_{V}(R C)\right\rangle+\mathrm{BH}$ & $4.3 \pm 0.9$ & $1.7 \pm 0.1$ & $3.9 \pm 1.0$ \\
4) $\left\langle M_{V}(R C)\right\rangle+\mathrm{SFD}$ & $6.3 \pm 1.3$ & $1.7 \pm 0.1$ & $6.0 \pm 1.7$ \\
\hline
\end{tabular}


This figure "Crowl.fig1.jpg" is available in "jpg" format from: http://arxiv.org/ps/astro-ph/0104227v1 


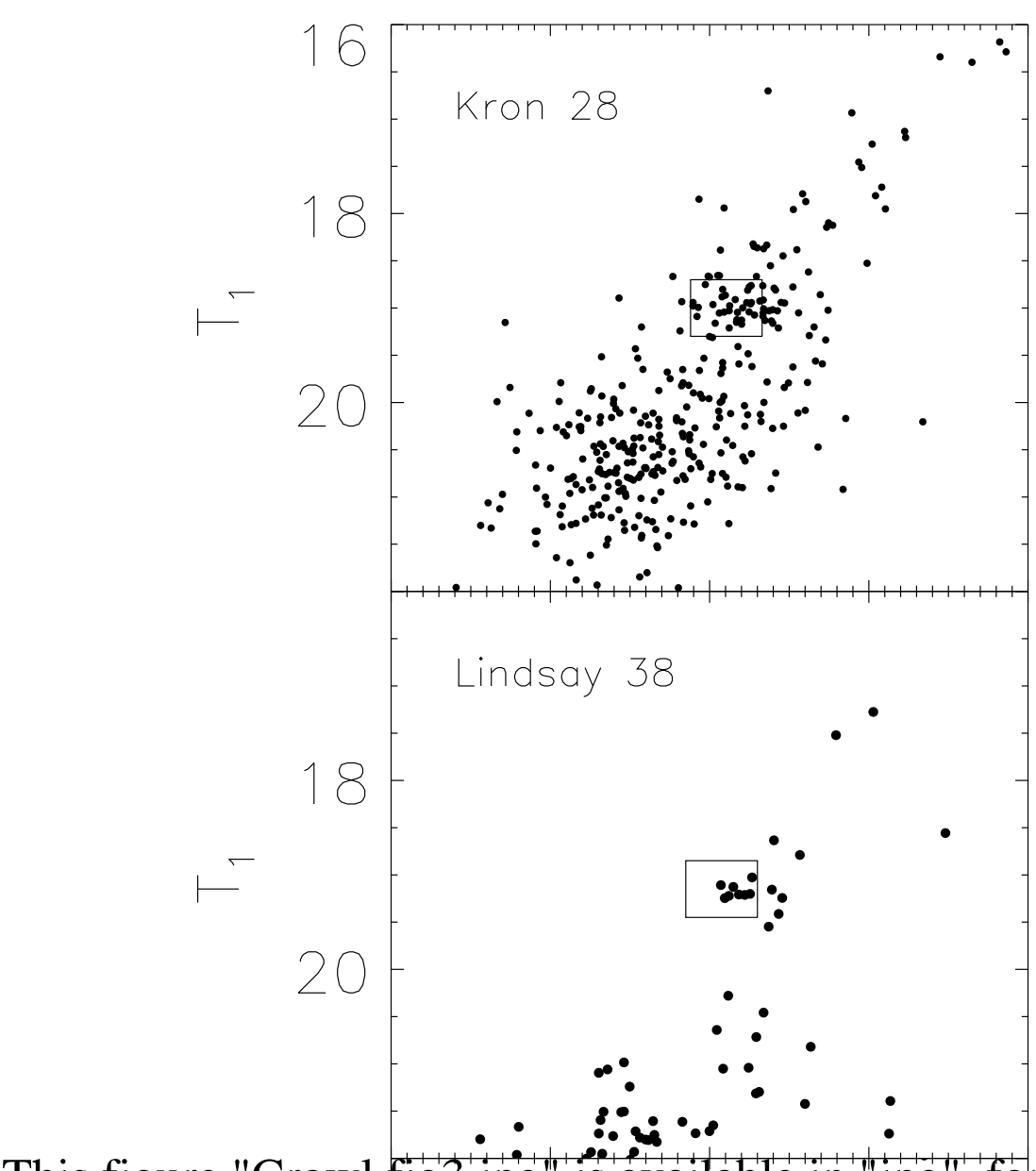

This figure "Crowl.fig3.jpg" is available in "jpg" format from:

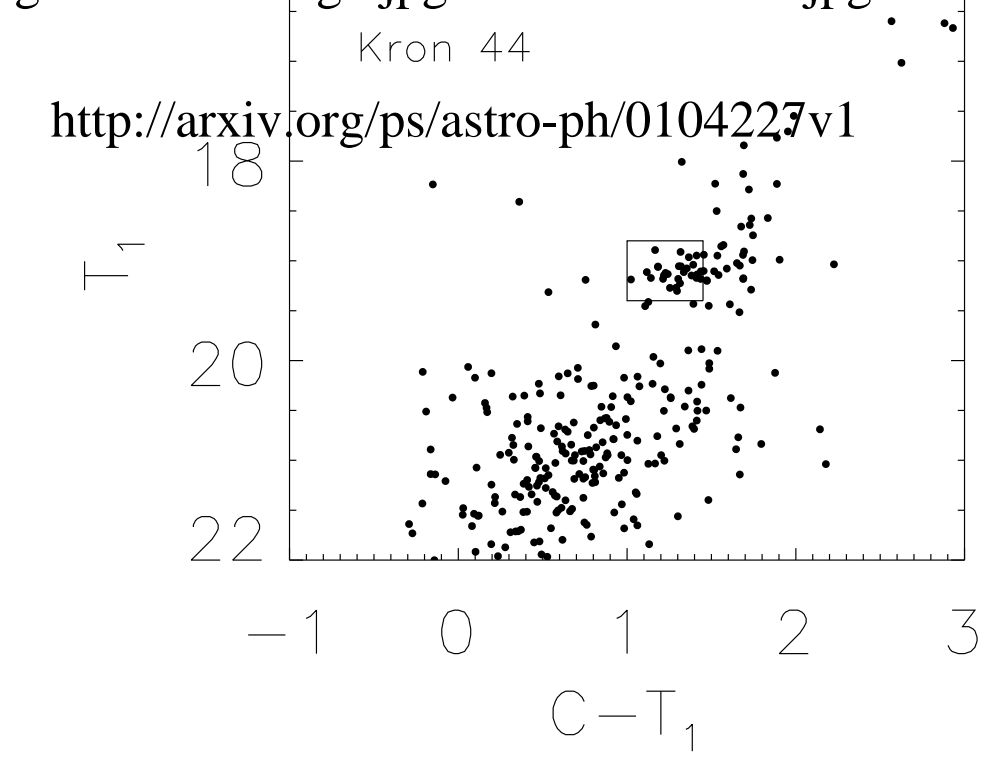


This figure "Crowl.fig4.jpg" is available in "jpg" format from: http://arxiv.org/ps/astro-ph/0104227v1 
This figure "Crowl.fig5.jpg" is available in "jpg" format from: http://arxiv.org/ps/astro-ph/0104227v1 


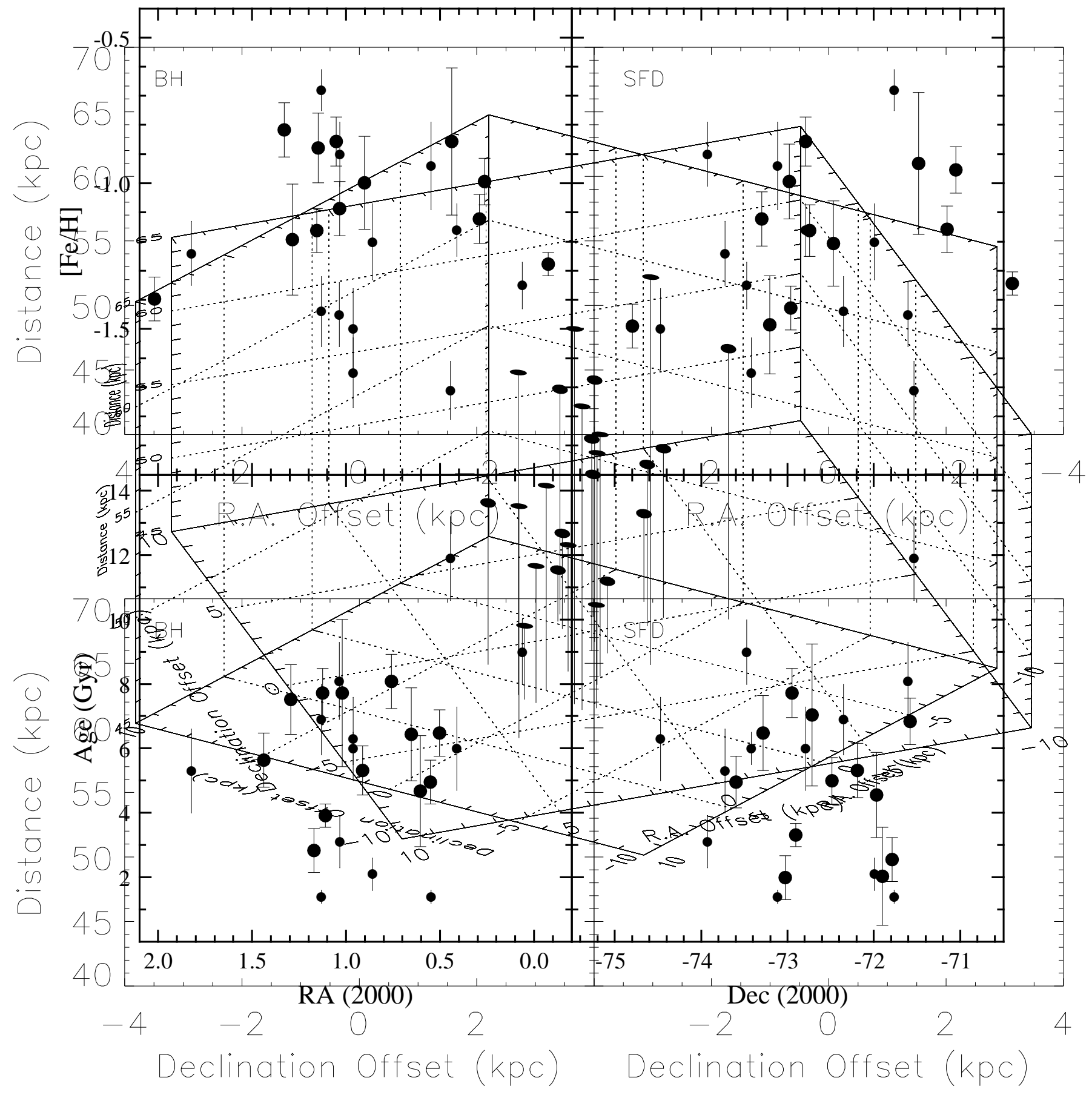

Fig. 8 - Crowl et al. 